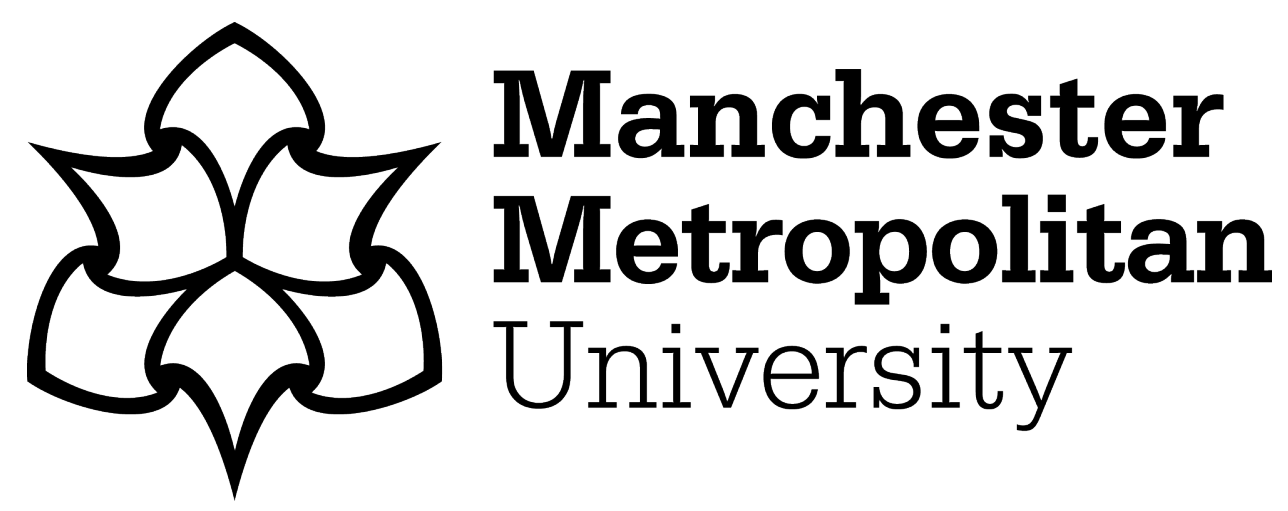

Melthis, John, Brown, Alex, Tang, Stephen and Hanneghan, Martin (2016) Using Serious Games to Create Awareness on Visual Impairments. In: 2015 International Conference on Developments of E-Systems Engineering (DeSE), 13 December 2015 - 14 December 2015, Duai, United Arab Emirates.

Downloaded from: https://e-space.mmu.ac.uk/623242/

Version: Accepted Version

Publisher: IEEE

DOI: https://doi.org/10.1109/dese.2015.65

Please cite the published version 
See discussions, stats, and author profiles for this publication at: https://www.researchgate.net/publication/308043052

\section{Using Serious Games to Create Awareness on Visual Impairments}

Conference Paper · December 2015

DOI: 10.1109/DeSE.2015.65

\section{CITATIONS}

0

4 authors, including:

John Henry

Manchester Metropolitan University

4 PUBLICATIONS 7 CITATIONS

SEE PROFILE

Martin B. Hanneghan

Liverpool John Moores University

43 PUBLICATIONS 404 CITATIONS

SEE PROFILE

\section{READS}

42

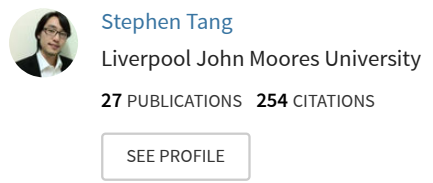

Some of the authors of this publication are also working on these related projects:

Combining the Internet of Things and Serious Games (Smart Serious Games) View project

Utilising the Third Dimension to Visualise Cyber Security Requirements in BPMN View project 


\title{
Using Serious Games to Create Awareness on Visual Impairments
}

\author{
John Melthis ${ }^{1}$, Alex Brown ${ }^{2}$, Stephen Tang ${ }^{3}$ and Martin Hanneghan ${ }^{4}$ \\ Department of Computer Science, \\ Liverpool John Moores University, \\ James Parsons Building, Byrom Street, Liverpool, L3 3AF, UK. \\ Email: j.melthis@2015.ljmu.ac.uk¹, alexbrown@malloc.co.uk ${ }^{2}$, o.t.tang@ljmu.ac.uk ${ }^{3}$,m.b.hanneghan@ljmu.ac.uk ${ }^{4}$
}

\begin{abstract}
Visual impairments define a wide spectrum of disabilities that vary in severity, from the need to wear glasses, to permanent loss of vision or blindness. This paper discusses the process undertaken in creating two simulators, one which emulates partially-sighted visual impairment and another focused on full-blindness. In order to create the simulators, extensive research was conducted surrounding the effects of partially-sightedness and blindness, highlighting existing software and games that promote awareness for visual impairments. This paper underlines the necessity of raising awareness for visual impairments and the effectiveness of applying serious games for this very goal. After developing the simulators, experiments were conducted to evaluate the effectiveness of it. Findings from the experiments were analysed and documented.
\end{abstract}

Keywords—Visual Impairments, Visual Impairment Simulators, Serious Games, Games for Awareness, Partial - Sightedness, Full Blindness.

\section{INTRODUCTION}

Visual impairments hinders one's vision by making it difficult to perceive visual information from their surroundings. Visual impairment can be inflicted or be present since birth. Some visual impairments can be corrected with prescription glasses or contact lenses [1]. The vision from other forms of more severe visual impairment such as macular degeneration, diabetic retinopathy [2] glaucoma [3], retinitis pigmentosa [4] and cataract [5], can only be restored through permanent solutions that require one to undergo a medical procedure. However, there are some forms of visual impairment such as retinopathy of prematurity and full visual impairment, caused by disease and age-related cell degeneration [6] that remain hard to treat.

Depending on the severity of the impairment, visually impaired individuals may experience segregation, due to the lack of understanding by the general public for their daily struggles, causing them to mask their disability in public. Some forms of awareness can be raised to help the general public understand the relative complications [7]. This project aims to raise awareness for individuals with visual impairments in order to reduce public segregation and increase empathy for these conditions. Visual impairments can hinder depth perception and spatial awareness and leave impaired individuals only seeing through one-eye, creating a smaller field of vision than an average person, which can lead to complications when in crowded environments.

In the $21^{\text {st }}$ century, computer gaming has emerged as a culture amongst the younger generation [8]. Computer games are now being repurposed for non-entertainment domains such as health, advertisement, education, science and research, modelling environments and visualisation. The nonentertainment applications of computer games technology are defined as "serious games". Many of these applications have received positive outcomes [9]. In addition, game technologies are also becoming further advanced, mainstream and accessible by the public. Technically able domain experts can now choose to develop their own game software using either royalty free game engines or open source game engines. By developing a serious game for the general public to experience multiple forms of visual impairments, awareness can be raised in a safe and interactive environment.

This paper investigates the application of game technologies for the development of two serious games that simulate the effect of visual impairments, one caused by a rare condition, namely retinopathy of prematurity and the second one blindness. Section II presents a review into existing computer games and applications that simulate the effects of visual impairment. Section III discusses the components of game technologies and the digital content creation tools that support the development of the simulators. The design and implementation of the visual impairment simulators are described in Section $I V$. The details and findings from the experiments are presented in Section V. Section VI concludes and presents future works recommended for this project.

\section{COMPUTER GAMES THAT SimUlate VisUAL IMPAIRMENTS}

Computer games are visually stimulating interactive content that engage users to take part in goal-directed play. Typical computer games showcase their unique visuals and often the visually impaired community are excluded from enjoying it. Nevertheless, there are a few computer games designed around the gameplay idea of using primarily auditory cues which can be enjoyed by anyone, including the visually impaired community, such as Blindside and The Nightjar.

Blindside [10] is a role-playing horror-adventure game that features extensive use of 3D audio in its game-play. Players are tasked to navigate (using keys and mouse on PC) their way 
around the city to a safety point by relying only on audio cues. Although Blindside is designed for entertainment purposes, it advertises itself as a serious game with strong emphasis on accessibility, with a focus for the visually impaired community.

Nightjar [11] is a role-playing mobile game where players use only their hearing to help navigate within a spacecraft called "Nightjar". Players respond to the game by tapping on the screen and control the avatar's movement using very a simple UI. The game simulates the effects of blindness and does not promote itself as a serious game. However, it does deliver subliminal messages that are found in edutainment, and highlight the success non-visual applications can have in the current market.

The Impairment Simulator Software [12] developed by the University of Cambridge, is a web-based tool accessible by the general public, to gain a better understanding of different vision and hearing impairments. Human Computer Interaction (HCI) students and practitioners, who need to understand the effects of the impairments, use it. The software features a vision simulator which mimics visual impairment conditions such as macular degeneration, diabetic retinopathy, glaucoma and retinitis pigmentosa. Users set the severity of the condition and see the effect on a still image, from a first person perspective. This tool is not aimed at promoting awareness for visual impairment, but nevertheless can be used as an educational tool.

The reviewed computer games are unique, as they require users to rely on their hearing to navigate. Though these games do not promote awareness about the challenges of visual impairment, they do feature messages on the importance of vision. There is no simulation available that could be used to re-create the effects of partial-sightedness, and there is no simulator which focuses on raising awareness for the blind. By taking advantage of games technology, the effects of partialsightedness and blindness can be simulated within a virtual environment and the serious game application could be used as a tool to generate awareness and educate the general public.

\section{GAME TECHNOLOGIES AND DIGITAL CONTENT SOURCING}

Computer games initially were programmed as a singular entity in native programming languages [13], an approach replaced with game engines. A game engine is a software library with components that perform game specific tasks such as rendering graphics (2D or $3 \mathrm{D})$, computing motion, collision detection, simulating intelligence, handling user's input and managing game data [14].

For simulating partial-sightedness and blindness in a 3D virtual environment, the following game engine components are pivotal to the development of the simulators:

- Renderer performs all the computations that generate both 2D and 3D imagery that composes the virtual environment and visual effects for the virtual world in the simulator.

- Game Physics facilitates the computation of motion, collision detection and collision response to mimic the physics in the physical world, which adds realism and simulates the effect of inability to accurately judge distance, when interacting with virtual objects within the virtual world.

- Audio provides the facility to playback sound effects to accentuate the experience of false spatial judgement when interacting with virtual objects in the simulator.
Modern game engines support the use of threedimensional sound via a set of $\mathrm{X}, \mathrm{Y}$ and $\mathrm{Z}$ coordinates, this creates a realistic representation of real-world sound.

- Lighting illuminates the virtual environment and adds depth to the $3 \mathrm{D}$ objects.

- User Interface (UI) provides the Graphical User Interface (GUI) components to ease the process of creating menus and present static information to the user. UI's are essential as they provide real-time data; such as a health percentage, in a visualised format for the user.

- Input maps the appropriate user's action via human interface devices such as keyboard and mouse to ingame actions to control the avatar's actions within the virtual world.

- Scripting enables the developer to utilise the game engine functionality and programme the flow and interaction of the visual impairment simulator without the need to change the game engine implementation.

- Level Editor provides a visual environment that allows developers to construct the virtual world and to alter the game-related parameters with ease.

Game engines only provide the technological solutions that enable game software to operate. Game software also requires content such as graphics, models, sounds and animation data, to provide them with the necessary visual and auditory representations. Game content can be produced using digital content creation tools, these tools specialise in different areas including 2D image manipulation (Photoshop), 3D mesh generation (3ds Max), animation (Motion Builder) and Audio development (Audacity). Alternatively, free or paid content, could be sourced from the web-marketplace such as TurboSquid or be developed via Autodesk Character Generator.

\section{DESIGN AND IMPLEMENTATION OF VISUAL IMPAIRMENT SIMULATORS}

The reviewed computer games and applications provide insight on game applications that simulate the effects of visual impairments. To simulate the effects of blindness, the use of $3 \mathrm{D}$ audio is vital, as it provides the direction of sound. For simulating visual impairment, 2D overlays and filters are used to disrupt a 3D virtual environment. Partial-sightedness, causes limited field of view and difficulty in perceiving depth and 3dimensional space accurately. In this section, the design and implementation of the simulators for partial-sightedness and blindness using modern game technology, are discussed.

\section{A. Requirements}

\section{1) Requirements for Partial-Sightedness Simulator}

A challenging real-world scenario for partially-sighted sufferers can be observed when the individual descends a glass staircase, similar to those installed in hotels or Apple stores. It presents a great challenge to these individuals to process "cropped" real-time visual information obtained from a single eye, resulting in refracted images, due to varying depth between the glass and air, which can lead to falls. This would be the ideal scenario for the partial-sightedness (PS) simulator.

The PS simulator's goal is to model a multi-storey glass staircase, highlighting the effect of a "cropped" field of view with the inability to accurately judge distance between objects within sight. This simulator aims to target $18-50$ year old audiences, with the assumption, most users within this age 
group live an active lifestyle and have most interaction with the public. It would be appropriate to use the PS simulator as a medium to educate the user group about the effects of partial visual impairment.

The PS simulator must be easy to use and provide a first person perspective. Clear instructions will be set out, and the controls introduced should be familiar to the user, reducing the learning curve and thus lowering the barrier when using the simulator. It is important that the PS simulator offers two modes: (1) normal mode and (2) impaired mode. The normal mode should allow the user to calibrate their senses to the virtual environment and the impaired mode should then virtually alter their perception, allowing them to experience the effects of partial-sightedness.

The PS simulator will be using the conventional first-person PC game controls (WASD or arrow to move and spacebar to increase the speed of movement) and the mouse to navigate the player in the virtual environment. The virtual camera will be placed at the eye level of the player's character. The player must be able to experience the jittery motion and the visual sense of losing balance when descending on the staircase. Audio will be used to maximise the user's experience, immersing the user into the $3 \mathrm{D}$ virtual setup. The virtual camera will be "shaken" to simulate the effect of loss of balance. The glass staircase should also be populated with static 3D objects that act as obstacles, presenting some form of challenge. In addition, some areas will be poorly lighten, adding complication to the already "cropped" field of view.

\section{2) Requirements for Full-blindess Simulator}

The full blindness (FB) simulator will place users in a road crossing scenario which, in the impaired mode, requires the user to rely solely on auditory cues to get from point A to point B. Projecting an individual who suffers from blindness does not require such specific conditions as the partial-sightedness requirements, due to the blind encountering problems more often with simple daily tasks. A simulator which renders the user's monitor black, and the objective of getting through a street will suffice as the setting for the FB simulator.

Within the FB simulator there will be signposts, which prompt on screen messages to alert users of their presence. Upon finishing the simulator, a final on-screen message will appear, providing two options whereby users can replay in normal mode or quit. During normal mode, users will have the ability to see within the $3 \mathrm{D}$ virtual environment. This aids users to evaluate the differences between normal sighted mode, and blindness mode.

The FB simulator will include moving vehicles on the roads with collision detection, which on contact, will cause the character to fail the simulation, prompting a message informing that they must restart the simulator. The vehicles are added to create a level of difficulty and realism, better replicating the difficulties imposed on individuals with blindness, in day to day life. Conventional first-person controls, applied to a keyboard and mouse, will also be utilised for this simulator, increasing familiarity for the participant.

\section{B. Methodology}

The PS and FB simulators were developed in three stages design, asset production and prototyping. This project adopted the rapid application development (RAD) methodology [15], allowing for the addition of features or functionality to the simulators. This methodology places less emphasis on activity planning and more emphasis on developmental activities. The scope and requirements of the simulators, were incrementally outlined throughout the project alongside development. The design and software underwent a number of iterative revisions, before the final versions were acknowledged and produced, defining the flow and feel of the applications.

Once the design of the visual impairment simulator had been finalized, production and sourcing of art assets for the PS simulator took place. Developed assets were created using low polygon techniques, whilst resembling the identity of the object in real-life, minimizing lag time in runtime.

High-quality assets were then imported, replacing the lowfidelity 3D models into the prototype. All 2D graphics were replaced with themed graphics to provide a unified look and feel for the simulators. Texture maps were used to enhance the virtual 3D environment. These maps include normal maps, which provide the illusion of bumpy surfaces, specular maps, for adding a shine effect to objects and gloss maps, which add a smooth lighting effect, to objects such as glass or marble. In the PS simulator, lights were tuned to provide the view of a poorly lighten environment. Collision effects, menus and additional player attributes were scripted within the simulators. The FB simulator also includes simple path animations scripted for the car movement. The final version of the prototypes will be tested for bugs before use in the planned experiment for evaluation (See Section V).

\section{Choice of technologies}

The development of the simulators can be categorised into two stages: (1) production of assets for the 3D virtual world and (2) implementing the simulators using a game engine. Both stages require different types of tools and technology that support the activities stated.

In this project, most of the custom 3D assets for the virtual world are produced using Autodesk 3DS Max. 3DS Max can export files in multiple formats, one of which is FBX. FBX file formats are compatible with UDK and Unity as valid meshes. Complex models such as 3D characters that are timeconsuming to model, are sourced from the web-based marketplace. Due to constraints (short development time-frame and zero-budget) on this project, only royalty-free and no-fee 3D assets are used. Adobe Photoshop produced the 2D static graphics such as user interfaces and textures, due its image editing efficiency. The textures were created by painting UV maps, which were produced in 3DS Max. Real-world images can be placed onto any UV map, creating a realistic effect. Different types of maps can also be created such as reflection, refraction, normal or specular.

For the PS simulator, the Unity Game Engine is chosen as it allows computer games to be published to multiple platforms such as Windows (PC), Mac, Android, iOS and the web where audiences can run interactive real-time $3 \mathrm{D}$ within the web browser using the Unity Web Player plugin. The tool is available for free to use for non-commercial PC and web deployment.

The FB simulator was developed using the Unreal Development Kit (UDK) with the default first-person view setup. The player controls the movement using the arrow or WASD keys, and the mouse is used to control virtual character's point of view and direction of motion. UDK's Matinee - an animation tool within UDK, was used to manipulate the animation paths of objects, and UDK's Kismet - visual scripting facility was used to script events and game play. The menu interface for the FB simulator was developed using Adobe Flash and integrated using Scaleform to output Flash content in the UDK environment. Both utilised game engines boast a large online support community, including email support and online forums in search for advice. 


\section{Implementation of Visual Impairment simulators}

Both, the PS and FB simulator, are designed to operate at two modes. The normal mode provides the correct view of the virtual world (see Figure 1 and Figure 2).

\section{1) Features implemented for PS Simulator}

In order to simulate the effects of partial-sightedness, imperfections such as limiting the field of view, altering perception of depth and simulating fatigue were programmed into the PS simulator.

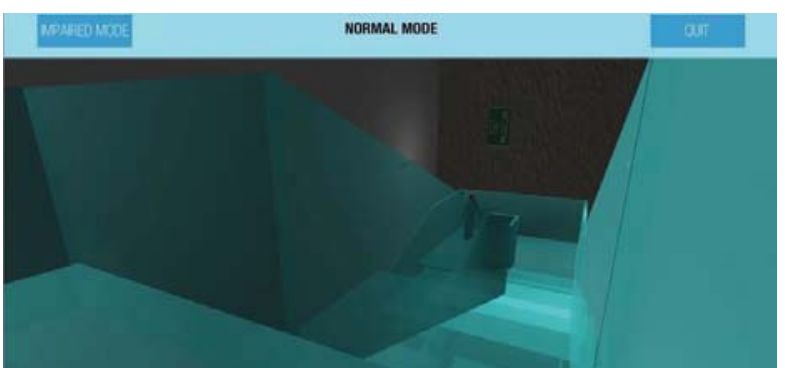

Figure 1: Screenshot of the PS simulator in normal mode (Source: Author's own collection)



Figure 2: Simulator in progress, playing in normal mode (Source: Author's own collection)

The horizontal field-of-view for normal sighted individuals is approximately $180-190^{\circ}$ with $120^{\circ}$ of overlapping area and $30^{\circ}$ to $35^{\circ}$ of monocular vision on each side of the eye [16]. The loss of sight from one eye would reduce the field of view by approximately $22 \%-26 \%$. To simulate the effect of the sight loss in the PS simulator, an opaque black filter was programmed on the left side of the user's display to reduce approximately $25 \%$ of the visual in the simulator (See Figure $3)$.

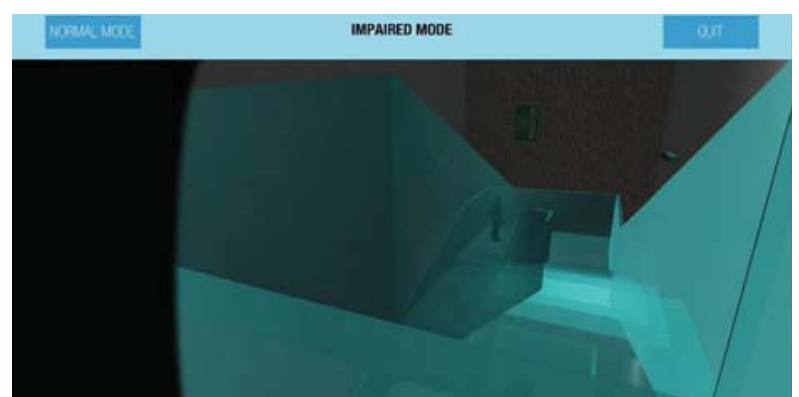

Figure 3: Screenshot of the PS simulator in impaired mode (Source: Author's own collection)

The slight gradient on the opaque filter applied is to mimic the "bokeh" effect (depth-of-field effect) experienced when the left-eye's vision is not present. The textures are created in Photoshop and used as a GUI Texture in Unity, which appears as an overlay in the impaired mode. The GUI texture is then positioned to the left of the screen to simulate the loss of sight in the left eye.

A normal-sighted individual possesses stereopsis vision which enables one to perceive depth and the 3-dimensional structure of an object. This ability is lost when an individual can only see through one eye, which results in monocular vision. In the $3 \mathrm{D}$ virtual world, the computer screen projects monocular vision. In order to exaggerate the inability to perceive accurate distances between objects, the rhythm of descending the stairs is broken. A rhythm is introduced whereby the user experience the same pattern of motion when descending the stairs, reinforced with the playback of "footstep" sound effects. An additional collision box that is raised from the surface of the glass staircase, to extend the depth of a step and not made visible to the user, is placed on each segment of the stairs (see Figure 4). This is done to break the rhythm and create an artificial sense of losing balance as a result of falsely judging distance between steps. This effect of losing balance is also reinforced with the playback of a "losing balance" sound effect (human shock), where each of the soundboxes are scripted to playback a sound file.

The default motion of descending the staircase is at approximately the average walk-speed. One of the programmed features allows users to increase the speed of descending the staircase. In a real world scenario, a partially sighted person may find the increase of speed overwhelming as the brain needs to process the limited visual information quickly, and calibrate with the motor system. Often one may experience fatigue when doing so. In order to simulate the fatigue effect, a scripted camera shake function was implemented to provide a jittery motion to the display.



Figure 4: Setup of additional collision boxes and sound effects in the impaired mode (Source: Author's own collection)

\section{2) Features implemented for FB Simulator}

The blindness mode in the FB simulator projects a black screen to simulate the loss of vision. There are two notifications on the top of the display; one notifying what the user needs to press to access the options menu, and the other informing the user that the simulator is running. When interacting with the FB simulator, the user will be prompted with messages, which overlay the black display to inform them of the current situation for ease of use. For example if the user walks beside a signpost a message is prompted instructing the user: "Press " $E$ " to use the signpost" (see Figure 5).

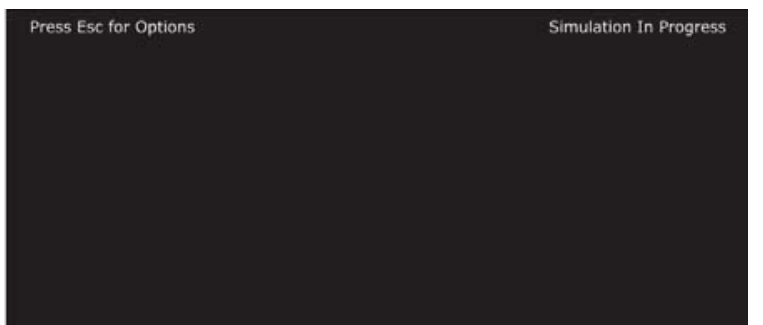

Figure 5 - Simulator in progress, playing in blind mode (Source: Author's own collection)

Since the primary goal of the FB simulator is to raise awareness for those who suffer from full visual impairments, developing realistic visuals is deemed redundant. More attention is placed on the use of sound. Cars within the simulator have no unique form and appear as a generic car chassis (see Figure 2). Within the FB simulator there are 3D sounds placed both on static and dynamic objects around the 
3D virtual world. These sounds will be the only form of input from the virtual world that users can rely on to achieve the objective in the FB simulator. Cars which patrol the simulator have different sounds attached to each one, enabling the user to identify multiple vehicles nearby. If the user collides with a car, the simulation ends and user will be prompted to restart the simulation again.

At the start of the simulation, users will be prompted with a message displaying the simulator's purpose and goal (to reach the subway). The subway is a visible building in the normal mode and has a train sound effect which is placed by the station, and is the crucial auditory cue for participants in the blindness mode. As the FB simulator has a larger map than the PS simulator, the character's walking speed is faster. The options menu allows users to view the controls, restart and exit the simulator.

\section{EVAluation of Visual Simulators}

Experiments were conducted to evaluate the effectiveness of the simulators, as a tool to promote awareness about the outlined visual impairments. Participant feedback was gathered using questionnaires, observation and interviews. Through the utilisation of multiple data gathering methods, cross validation of data can be performed, therefore improving the integrity of findings for this study.

\section{A. Experiments Setup}

The experiments were carried out in a computer lab with a Four PC setup to be used by participants during the experiment. The first PC (with headphones) was used to run the PS simulator, with the second, used to gather pre and post simulation questionnaires using an online questionnaire tool (surveymonkey.com). A third PC was used to record findings from interview and observation during the experiment. The fourth $\mathrm{PC}$ of the experiment ran the FB simulator.

Participants of the experiments were Liverpool John Moores University (LJMU) students who expressed interest through a 'call-for-participation' email or approached randomly on the day of the experiment. Each of the participants were given a short brief about the experiment and the process they will undergo. The participants were first required to complete the pre-simulation questionnaire consisting of six questions to gather demographic information. They were then given brief instructions for the use of the simulator. Participants' begin the simulation with the normal mode and move on towards the impaired mode whenever ready. The simulation session lasted approximately 5 to 10 minutes depending on the participant's ability to navigate around the $3 \mathrm{D}$ virtual environment and if they choose to go through the simulation multiple times. Participants were individually observed and notes regarding their interaction and response were recorded. Upon completion of the simulation, participants were asked to complete the postsimulation questionnaire containing nine questions that record the participant's experience with the simulator. Some of the participants were interviewed informally on a one-to-one basis, gathering further details on their experience with the simulator. Each participant spent no longer than 15 minutes collectively. Both the PS and FB simulators follow the same experiment format.

\section{B. Findings and Analysis}

Both the experiments were conducted once a week (on the same day) for the duration of two weeks with each session lasting for approximately 2 hours.

\section{1) PS Simulator Experiment outcomes and Analysis}

A total of 20 participants took part in the PS simulator experiment with a split of 35\% (7) female and 65\% (13) male.
However, only 19 participants completed the post-simulation survey. Out of the 20 participants, $85 \%$ (17) were aged 18 to 25 and the remaining $15 \%$ (3) were mature participants (26 to 50). Amongst the participants, 25\% (5) of the participants suffer some form of visual impairment and only 1 out of the 5 experienced some form of discrimination. When asked about the degree of awareness and the effects of visual impairment has on an individual, $20 \%$ (4) responded that they are very aware of it, 35\% (7) are aware while the remaining 45\% (9) have little awareness on how the condition would affect one.

The data from the PS simulator experiment suggested that the awareness of the effects of visual impairment is affected by one's age and visual ability, with participants with no visual impairment indicating minimal awareness for the conditions, concluding younger persons and those who are not affected by such impairments are more likely to have little awareness compared to the contrary.

All participants from the PS simulator experiment rated themselves good to excellent in terms of competency with computing and therefore should have little issue when using the simulator. However, $21.05 \%$ (4) of the 19 participants from the PS simulator experiment responded that they find it difficult navigating within the virtual $3 \mathrm{D}$ environment. It was then discovered that these participants did not play PC games and felt unfamiliar with the control setup used. The usability of the simulators was unaffected, this is evident when most participants (18) responded in the post-simulation questionnaire that the simulator was either "easy" (52.63\%: 10) or "very easy" (42.11\%:8) to use.

During the PS simulation, participants were asked to navigate in both the normal mode and impaired mode. All participants (19) felt it was either "easy" (52.63\%: 10) or "very easy" (47.37\%: 9) to navigate the avatar down the glassstaircase. In the impaired mode, the results shifted towards the "easy" (63.16\%: 12) and "moderate" (31.58\%: 6) range. Only 1 of the participants $(5.26 \%)$, found it "very easy" to complete the task in the impaired mode. One of the notable behaviours observed during the simulation is participant's tendency to lean towards the wall-side when navigating avatar down the glassstaircase during the impaired mode that simulate loss of the left eye's vision. One of the participants stated in the interview that he was unaware of such behaviour and only upon reflection that he realized it was a subconscious decision. This is a natural form of behaviour for the visually impaired to make use of static-objects in the surroundings as aid to navigate around. The sound effect of "surprise" did also affect some of the participants. A participant mentioned in the interview that she felt slightly uneasy, as if it was an additional challenge that she had to overcome to maintain focus and complete the task. These indications suggest the PS simulator was able to simulate some of the effects of partially sighted visual impairment.

The result from the PS experiment is encouraging. Prior to using the PS simulator, 45\% (9) of the participants had "little awareness" about the condition whereas 35\% (7) were "aware" of it and $25 \%$ (5) were "very aware" because they have some form of visual impairment. After using the simulator, 20\% (4) of the participants have converted from having "little awareness" to "aware" of the effects of partial sighted visual impairment. Although, the simulator is not able replicate the full effects of partial-sightedness, it has been a useful tool in generating awareness. All participants (19) were able to identify the type of disability and the purpose of the simulation in the post-questionnaire. They also believe that it is important to raise awareness about the effects that partial visual impairment have on individuals. 


\section{2) FB Simulator Experiment outcomes and Analysis}

In the FB simulator experiment, 17 participants took part in the experiment; 71\% (12) male and 29\% (5) female. All 17 participants completed the post-simulation survey. 94.12\% (16) of the participants for the FB simulator experiment aged between 17 to 26 years old and one aged between 27 to 36 years old. When participants were asked if they understand the condition and difficulties of those who suffer with full blindness, 41.18\% (7) "strongly agree", whereas 58.82\% (10) replied they "agree". The responses received are expected as blindness is usually discussed in family and school settings, although the $58.82 \%$ (10) that agreed, shows further awareness can be raised for blindness. When participants were asked if they would be inclined to helping a blind individual, $11.76 \%$ (2) responded with no whereas $88.24 \%$ (15) chose 'yes', indicating empathy towards those who are blind.

Likewise, participants in the FB simulator were asked to navigate in the $3 \mathrm{D}$ virtual world in both normal mode and blindness mode in order to complete the given objective (to reach the subway station). Upon completing the FB simulation, no participant found the simulator "easy", however $23.53 \%$ (4) of participants developed relative comfort with it. $35.29 \%$ (6) of participants did not adapt well to not using vision, whereas $41.18 \%$ (7) managed to comprehend little interaction. Participants documented they found it easier to manoeuvre themselves whilst closing their own eyes, to focus better on what they could hear. This result is expected because all participants have good vision and have been relying on visual cues for navigation. By removing the reliance on visual cues in the blindness mode, participants had to rely solely on the auditory cues and process the limited information about the environment. This is proven challenging for most participants and it evidenced that the FB simulator did present similar challenges for those who lost their vision, in a controlled and safe environment.

Results from the FB simulator also proved the effectiveness of the solution as $35.29 \%$ (6) of participants felt they were vastly more aware of those who suffer from blindness after using the simulator, and 52.94\% (9) felt more aware, but lacked on audio queues, causing a fragmented experience. One participant felt FB simulator was not very informative and did not feel they gained any further insight into the effects of blindness. These results do highlight necessary further works but underline the potential for this application of serious games as $41.18 \%$ (7) of participants "agreed" and $58.82 \%$ (10) "strongly agreed" that the FB simulator did successfully portray the challenges that a blind individual would face in the given scenario. Participants were also asked if they became more aware when driving near a blind individual. $12.5 \%$ (2) stated that they felt no different where the remaining stated a change in their driving behaviour as they felt more alert. These answers reinforce an understanding among participants towards blindness.

\section{CONCUSIONS AND FUTURE WORKS}

In this paper, game technology has been utilised for serious games to raise the awareness for individuals that suffer from partial-sightedness and blindness. Scenarios that highlight the difficulties faced by sufferers of the two types of visual impairment conditions were created to allow normal-sighted individuals to experience the effects of visual impairments in a safe setting. Although the findings indicate that the experience was not fully immersive, it provides beneficial tools to convey the awareness messages to the audiences.

Furthering the research in this area, a plan to extend the PS simulator to run on the Oculus Rift and assess whether it would provide amplification to the engineered effects of partially- sightedness may benefit future prospects. The FB simulator could also be improved with the use of clearer and dynamic sound effects with greater variety, to make the auditory in the $3 \mathrm{D}$ virtual environment more realistic.

Both serious games would also benefit by becoming a single application. In addition, other forms of visual impairment could also be added to the application to allow users to experience it. Generating more awareness amongst the general public could help create communities that are inclusive and help sufferers of visual impairment live a better quality life and reduce segregation. This research could aid the scientific community also to pave the way for the future of visualassisting technologies, such as visual augmentations and other assistive devices. These assistive devices could help rehabilitate those who have recently suffered from a serious visual impairment and direct them towards leading a normal lifestyle with their disability.

\section{REFERENCES}

[1] N. R. Galloway, W. M. K. Amoaku, P. H. Galloway, and A. C. Browning, Common Eye Diseases and their Management. Springer Science \& Business Media, 2006.

[2] K. Shotliff and G. Duncan, "Diabetic retinopathy: summary of grading and management criteria," Pract. Diabetes Int., vol. 23, no. 9, pp. 418420, Nov. 2006

[3] T. Kersey, C. I. Clement, P. Bloom, and M. F. Cordeiro, "New trends in glaucoma risk, diagnosis \& management.," Indian J. Med. Res., vol. 137, no. 4, pp. 659-68, Apr. 2013.

[4] M. Kalloniatis and E. L. Fletcher, "Retinitis pigmentosa: understanding the clinical presentation, mechanisms and treatment options," Clin. Exp. Optom., vol. 87, no. 2, pp. 65-80, Mar. 2004.

[5] J. C. Javitt, F. Wang, and S. K. West, "Blindness due to cataract: epidemiology and prevention.," Annu. Rev. Public Health, vol. 17, no. 1 1, pp. 159-177, 1996.

[6] S. Resnikoff, D. Pascolini, S. P. Mariotti, and G. P. Pokharel, "Global magnitude of visual impairment caused by uncorrected refractive errors in 2004," Bull. World Health Organ., vol. 86, no. 1, pp. 63-70, 2004.

[7] N. Buys and J. Lopez, "Experience of Monocular Vision in Australia," J. Vis. Impair. Blind., vol. 98, no. 9, pp. 1-28, 2004.

[8] C. Pearce, "Productive Play: Game Culture From the Bottom Up," Games Cult., vol. 1, no. 1, pp. 17-24, Jan. 2006.

[9] M. Zyda, "From visual simulation to virtual reality to games," Computer (Long. Beach. Calif)., vol. 38, no. 9, pp. 25-32, Sep. 2005.

[10] "BlindSide - The Audio Adventure Game." [Online]. Available: http://www.blindsidegame.com/. [Accessed: 07-Jul-2015].

[11] The Nightjar on the App Store on iTunes." [Online]. Available: https://itunes.apple.com/gb/app/the-nightjar/id431598741?mt=8. [Accessed: 07-Jul-2015].

[12] "Impairment simulator software - Inclusive design tools." [Online]. Available:

http://www.inclusivedesigntoolkit.com/betterdesign2/simsoftware/simso ftware.html. [Accessed: 07-Jul-2015].

[13] L. Bishop, D. Eberly, T. Whitted, M. Finch, and M. Shantz, "Designing a PC game engine,” IEEE Comput. Graph. Appl., vol. 18, no. 1, pp. 4653, 1998.

[14] S. Tang, M. Hanneghan, and C. Carter, "A Platform Independent Game Technology Model for Model Driven Serious Games Development.," Electron. J. e-Learning, vol. 11, no. 1, pp. 61-79, Jan. 2013.

[15] J. Watkins, Agile Testing: How to Succeed in an Extreme Testing Environment. Cambridge University Press, 2009.

[16] C. G. Healey and A. P. Sawant, "On the limits of resolution and visual angle in visualization," ACM Trans. Appl. Percept., vol. 9, no. 4, pp. 121, Oct. 2012. 